\title{
The coccygeal pressure ulcer-does coccygectomy prevent recurrence?
}

\author{
Christian Huber $^{1} \cdot$ Roland de Roche $^{1} \cdot$ Carmen Rinaldo $^{1} \cdot$ Margret Hund-Georgiadis $^{1} \cdot$ Gerrolt Nico Jukema $^{2}$
}

Received: 20 November 2019 / Accepted: 25 May 2020

(c) The Author(s), under exclusive licence to International Spinal Cord Society 2020

\begin{abstract}
Study design This is a retrospective, non-randomized cohort study, with data collected during the regular annual visits between 2001 and 2019.

Objectives The aim of this study was to evaluate the efficacy of coccygectomy for coccygeal pressure ulcers in individuals with paraplegia due to spinal cord injury or other neurological causes and to evaluate its role in the prophylaxis of ulcer recurrence.

Settings This study included inpatients and outpatients with a coccygeal pressure ulcer who were treated surgically at our Institution REHAB Basel and were followed with regular annual check-ups.

Methods Individuals with category 3 or 4 acute or chronic coccygeal pressure ulcer (classification according European Pressure Ulcer Advisory Panel (EPUAP)) received coccygectomy in addition to rotation flap surgery. The operative care was provided exclusively by the head of the plastic surgery department at REHAB Basel. Standardized follow-up treatment was carried out according to the "Basel Decubitus Concept" and thus allowed continuous and usually lifelong, regular followup care.

Results Forty-nine individuals underwent coccygectomy from 2001 to 2019 due to coccygeal category 3 or 4 pressure ulcers. The observation period was between 1.5 and 18.3 years. In $86 \%$ of the individuals, no relapse occurred during the first year. Over the next 5 years $78 \%$ remained relapse free.

Conclusions In coccygeal pressure ulcer category 3 or 4, coccygectomy, in addition to sufficient rotation flap surgery, is a suitable method for recurrence prevention of pressure ulcer in this anatomic area.
\end{abstract}

\section{Introduction}

During the early acute or initial treatment of acute spinal cord injury (SCI), pressure ulcers often occur in the sacral or coccygeal region [1]. Every pressure ulcer in patients with SCI, regardless of localization or etiology, represents a significant and immediate reduction in quality of life for the person affected. In addition, the risk of pressure ulcer for those affected may last a lifetime [2, 3]. Besides urological and respiratory infections, pressure ulcers are the most common reason for rehospitalization and overall the second most common complication in individuals with SCI $[2,3]$.

Christian Huber

ch.huber@rehab.ch

REHAB Basel, Basel, Switzerland

2 University Hospital Zurich, Zurich, Switzerland
Long hospitalizations result in a decrease in quality of life. In addition, there is a cost of around CHF 100,000 per pressure ulcer in Switzerland [3]. In the United States, a sum of $\sim \$ 11$ billion is spent in 1 year for the treatment of decubitus ulcers. Pressure ulcers represent the highest cost factor among the complications in individuals with a spinal cord lesion $[4,5]$.

The coccygeal pressure lesion discussed in this work is a rare complication but an extremely important site for the individuals with tetraplegia and for individuals with high paraplegia [6] compared with other localizations [7-10].

In terms of pathophysiology, a coccygeal pressure ulcer should not be confused with recumbent sacral ulcers [11]. Coccygeal pressure ulcers mainly occur with extended use of the so-called "beach-chair" position i.e., sitting with a dorsally tilted pelvis in individuals with tetraplegia (Figs. 1-3) and thus an increased pressure load on the skin in the coccygeal region. Even with meticulous and consistent pressure ulcer prophylaxis, relapse is possible. In 


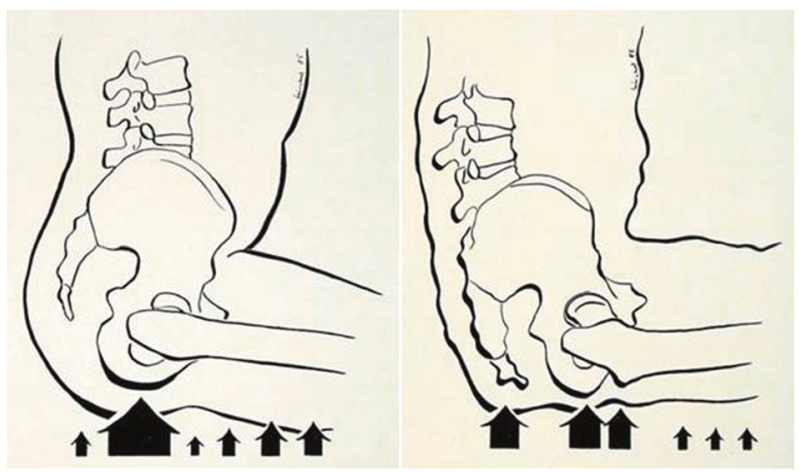

Fig. 1 Pressure distribution in different sitting positions [24]. First picture shows the pressure load on the ischium in an "normal" upright sitting position. Coccygeal pressure occurs with a dorsally tiltet pelvis (second picture) in a so called "beach- chair" position or in individuals with tetraplegia.

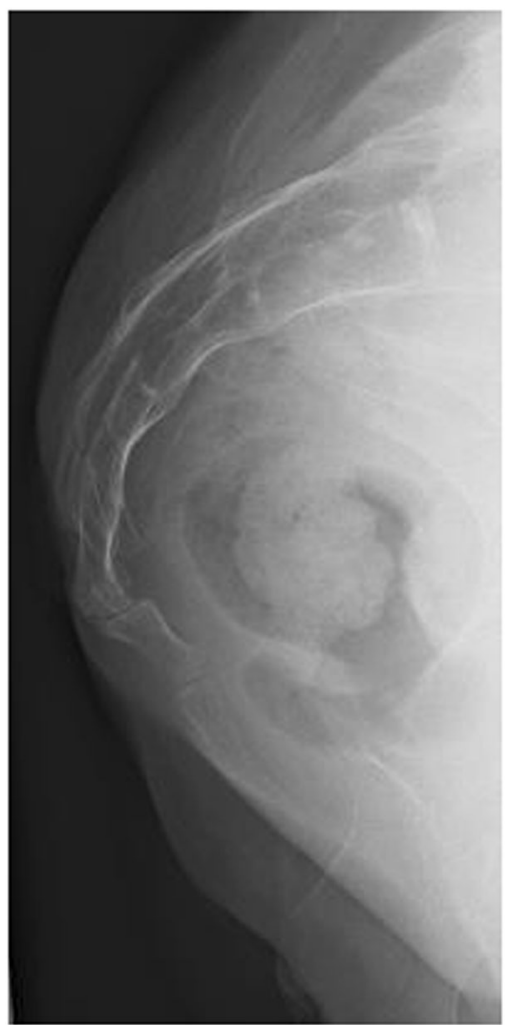

Fig. 2 Radiographic and palpatory instability of the Os Coccygeum in an individual with an incomplete paraplegia with chronic, recurrent coccygeal pressure ulcer.

contrast, sacral ulcers develop when lying down for too long without release. This is also due to the fact that the sacrum is a rigid bony block with a special bony anatomy in contrast to the intercoccygeal joints of the coccyx. Distinguishing the cause of a pressure ulcer is of utmost importance for therapy, care, and prevention [6].

Although the problem of a coccygeal pressure ulcer is of great interest to individuals with paraplegia as well as to

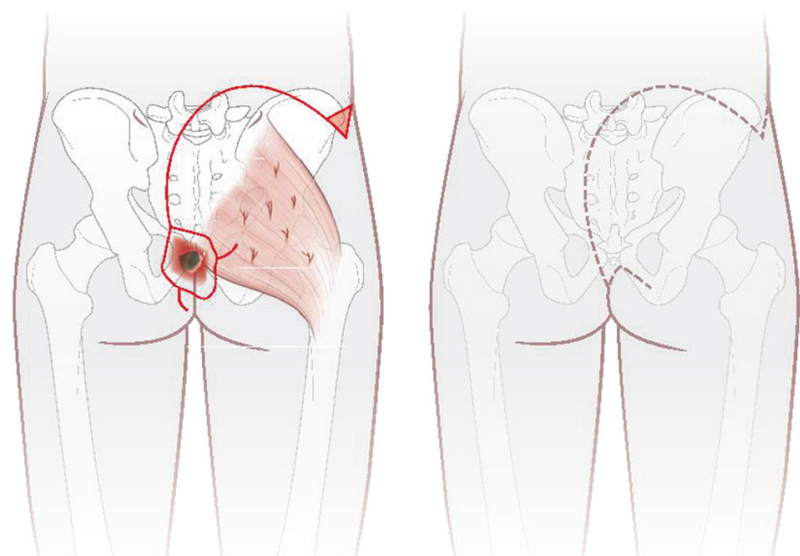

Fig. 3 Fasciocutaneous gluteal rotation flap (8). The unilateral mobilisation of the fasciocutaneous flap is a possibility to provide a tension free closure of a coccygeal pressure ulcer. The perfusion is supplied from perforating vessels coming from the deep braches of the superior gluteal artery and inferior gluteal artery.

rehabilitation professionals, there is paucity in the literature on the subject. Topics such as coccygodynia, first described in 1859 , are frequently found. Coccygodynia is also treated operatively by coccygectomy with good results [12-16]. Wound healing is considered to be problematic even in elective surgical coccygectomy and is associated with a recurrence risk of 30\% [15-19]. In the case of chronic recurrent pressure ulcer, the additionally existing problematic and compromising soft-tissue factors should be mentioned here with particular emphasis. There is a high risk of irritation in this often heavily loaded and scarred soft-tissue area. Pre-existing co-morbidities of individuals with SCI, multiple previous operations, and a wound area with a chronic pressure load also lead to a high risk of recurrence.

The aim of this retrospective study is to evaluate the efficacy of coccygectomy on recurrence and prevention of coccygeal pressure ulcer in individuals with SCI or other neurological causes of paralysis.

\section{Methods}

\section{Design}

This is a retrospective non-randomized cohort study; the data collection took place during the regular annual ambulatory visits between 2001 and 2019.

\section{Study population}

Inpatients and outpatients with category 3 or 4 coccygeal pressure ulcer who received flap surgery and coccygectomy 
between 2001 and 2019 were included in the study. Only individuals with a signed informed consent for data collection for research purposes were included. The coccygectomy was followed by continuous outpatient care in this patient group to minimize the high risk of relapses. This ensures a correct immediate treatment to prevent additional surgery.

\section{Study setting}

In the context of standardized pressure ulcer diagnostics, all pressure point lesions in the coccygeal area were routinely inspected with palpation and acute bony prominence (ABP) and subluxation were ruled out. Ligamentous instability of the os coccygeum, a past fracture, an existing $\mathrm{ABP}$, and pelvic alignment were taken into account in deciding further therapy. In the radiographic evaluation, the bony conditions and possible instability or dislocation were investigated $[6,18]$.

According to guidelines given by Provodin et al. [12] bone resection was applied as the most important surgical procedure for coccygeal pressure ulcer throughout the retrospective study period. This allowed a retrospective evaluation of the treatment itself, complications, and recurrence.

All procedures were carried out exclusively by one examiner to ensure an equal surgical routine. The surgical settings and method did not alter during the evaluation period nor did the setting of the rehabilitation regime [7]. After the initial necrectomy of total pressure ulcer, the coccygectomy was performed at the level of the sacral hiatus. The coccygeal bone was completely resected with the distal parts of the sacrum and with the cornua sacralis. In order to avoid a postoperative hematoma, a subperiosteal bone enucleation and closure of the periosteal sheath were performed. For tension-free occlusion, a Z-plasty, rhomboidal flap, or a gluteal fasciocutaneous rotationadvancement flap, was prepared and inserted. This gluteal fasciocutaneous rotation flap was mobilized unilaterally whenever possible as the other side can also act as a reserve during relapse. In addition to perforating vessels, the cranial half of the fasciocutaneous flap is supplied from the deep branches of the superior gluteal artery, and the caudal half from the inferior gluteal artery, all of which penetrate the gluteus maximus muscle. In order to minimize the tension in the area of adaptation, the semicircular incision is made far below the posterior iliac spine and above the trochanter. Thus, a rotating advancement of the flap on the defect zone and a subsequent tension-free suture can be provided. The flap tip was placed across the midline. This prevented repressurization of scar tissue in the previous wound area [7].

Post-coccygectomy care for all individuals was done according to the "Basel Decubitus Concept." Four weeks of bed rest with strict positioning regimens was followed by a subsequent remobilization step-by-step [20-22]. A 4-day postoperative break in low molecular weight heparinization also prevents bleeding in the remaining periosteum or under the flap plasty.

To analyze the effectiveness of coccygectomy, descriptive statistics were used for categorical and continuous data.

\section{Results}

In the rehabilitation hospital, a total of 374 surgeries, initial and repeat pressure ulcer surgeries, were performed from 2001 to April 2019. Of these, 210 procedures were performed in the ischial area, 69 in the sacral area, 30 for trochanteric lesions, and 65 for coccygeal pressure ulcers. In total 236 surgeries in individuals with paraplegia and 79 in individuals with tetraplegia were performed. Individuals with a major underlying neurological disease, such as multiple sclerosis, Parkinson's disease, or craniocerebral trauma had to be treated with surgery 59 times (Fig. 4).

In total, 52 individuals with SCI or neurologic disorders resulting in paraplegia or tetraplegia were operated on at least once between 2001 and 2019 due to coccygeal pressure ulcer. A general informed consent for research was available from 49 of these individuals. Of these $(n=49) 13$ were individuals with tetraplegia and 29 individuals with paraplegia (Table 1). Seven individuals had a primary neurological disease i.e., multiple sclerosis, Parkinson's disease, or neurotraumatic injury. Further characteristics of the individuals can be found in Table 1 .

Mean age was $54.14 \pm 16.52$ years (Table 1 ). The age distribution of individuals with coccygeal pressure ulcer $(n=49)$ shows a bimodal distribution at ages 50-54 and 65-69 years (Table 1). The follow-up period averaged 9.38 years (min./max.1.5-18.3 years).

In the first year after coccygectomy, four individuals (one with paraplegia, two with tetraplegia, and one with a neurological disease) had one recurrence.

Three individuals (one with paraplegia and two with tetraplegia) had two recurrences. These recurrences required surgical revision. In the other 42 individuals $(86 \%)$ of the

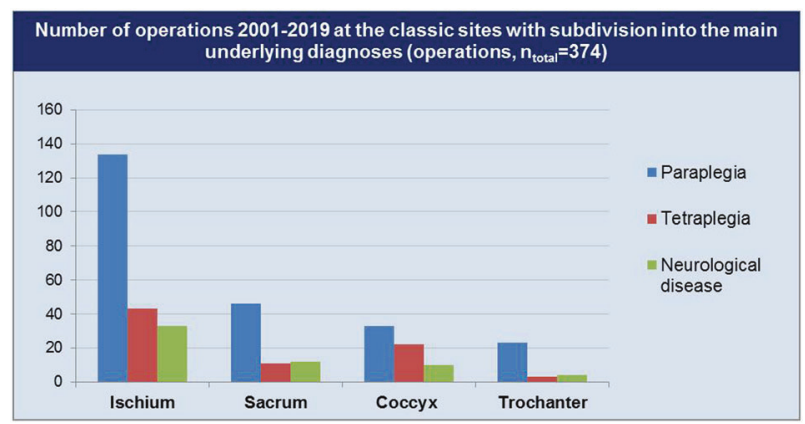

Fig. 4 Number of operations from 2001 to 2019 at the classic sites with subdivision into the main underlying diagnoses. 
Table 1 Characteristics of individuals at baseline, after 1 and 5 years after coccygectomy.

\begin{tabular}{ll}
\hline Number of individuals at baseline & 49 \\
\hline Mean age at coccygetcomy \pm SD [years] & $54.14 \pm 16.52$ \\
Gender & 23 female \\
& 26 male \\
& 20 AIS A (15 paraplegia, 5 tetraplegia) \\
Primary diagnoses & 11 AIS B (6 paraplegia, 5 tetraplegia) \\
& 9 AIS C (6 paraplegia, 3 tetraplegia) \\
& 2 AIS D (2 paraplegia) \\
& 7 neurological disease \\
& 49 \\
Number of individuals after 1 year after & 0 revision in 42 individuals \\
coccygectomy & 1 revision in 4 individuals \\
Number of revisions in individuals 1 year after & 2 revisions in 3 individuals \\
coccygectomy & 32 \\
& 8 individuals have not yet completed the 5 years \\
Number of individuals after 5 years after & follow-up period \\
coccygectomy & 9 individuals died because of other causes than \\
& surgical complications \\
& 0 revision in 25 individuals \\
& 1 revision in 4 individuals \\
& 2 revisions in 3 individuals \\
coccygectomy & $9.38 \pm 4.49$ \\
\hline
\end{tabular}

total population, there were no complications 1 year after coccygectomy.

Five years after coccygectomy, data of 32 individuals were available for analysis because nine individuals had died and eight individuals were $<5$ years post coccygectomy. Therefore, they did not complete the control period and could not be included. The individuals who died, did not die due to the surgical procedure nor due to surgical complications of the coccygectomy. Of the 32 individuals, four $(13 \%)$ had to be surgically revised due to one recurrence within five years after coccygectomy. Three individuals (9\%) suffered a second recurrence. In total, 25 individuals $(78 \%)$ were free of recurrences (Figs. 5 and 6) 5 years after the coccygectomy.

\section{Discussion}

There is a paucity in the literature on coccygeal pressure ulcer and its surgical treatment in individuals with paraplegia. Provodin et al. [12] reviewed 38 individuals who underwent surgery because of a pressure ulcer above the coccyx. The study group consisted of 15 (39.5\%) women and $23(60.5 \%)$ men. Four of the individuals $(11 \%)$ had initial presentation of pressure ulcer, 34 (89\%) had a chronic or recurrent pressure ulcer.

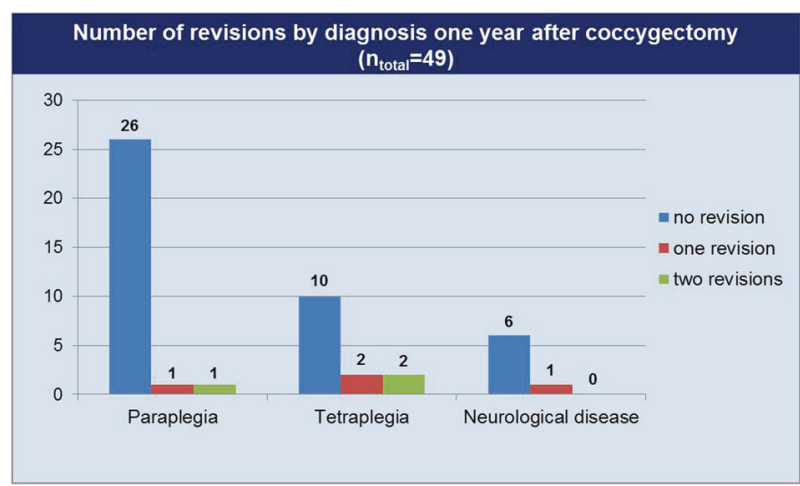

Fig. 5 Number of revisions by diagnosis.

Of the 38 operated individuals, only 6 individuals, those who did not receive initial coccygectomy, relapsed above the level of the coccyx. After relapse, these six individuals underwent coccygectomy and surgery. On the basis of these results, bone resection was considered to be the most important surgical procedure. Based upon this publication, we treated the individuals with a coccygeal pressure ulcer in our institution similarly (2001-2019).

Wound healing is problematic even in elective surgical coccygectomy and is associated with a recurrence risk of $30 \%$ even without any co-morbidities [15-19]. 
Number of revisions by diagnosis five years after coccygectomy $\left(\mathrm{n}_{\text {total }}=32\right)$

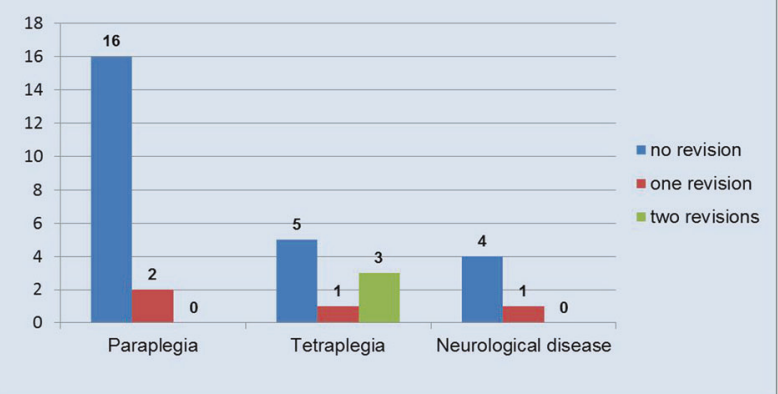

Fig. 6 Number of revisions by diagnosis 5 years after coccygectomie $(n=32)$.

In individuals with pressure ulcer caused due to an often chronically compromised soft-tissue factor, there is a high risk of irritation in this heavily loaded and scarred softtissue area. Pre-existing co-morbidities of individuals with SCI, previous surgeries, and a wound area with a chronic pressure load also lead to a high risk of recurrence. Previous studies have shown that level of SCI seems not to be associated with the occurrence of a coccygeal pressure ulcer [6] and this is similar to our findings.

The general risk of getting a pressure ulcer, especially in elderly people with an SCI, is very high. In a publication from 1994 by Langauer [23] the incidence of a hospitalization because of a pressure ulcer for a patient with an SCI is every third year. This indicates the fragility and the necessity of a continuous specific patient check especially for patients with a SCI.

Due to the risk of a recurrence in this patient group, we previously reviewed at the recurrences in all flap surgeries in the pelvic region [8]. In the time period from 2001 to 2010 we had 254 surgeries performed for 182 pressure ulcers. Thirty seven percent had minor complications, fourteen percent had major complications requiring repeat surgery. In total, $19 \%$ of repeat surgery within 10 years [8].

In the group of the coccygeal pressure ulcer we had $29 \%$ minor complications and $29 \%$ major complications, which required repeat surgery. This complication rate is similar to other literature [15-19].

In addition, we have $22 \%$ recurrence rate in total, which is similar to the other findings and is a little higher than in the other areas of the pelvis (14\%). This shows the high risk of recurrences in the coccygeal area compared with pressure ulcers in other regions of the pelvis. Since we can find up to $30 \%$ recurrence rate in the literature for individuals with no SCI or any co-morbidities [15-19], the coccygectomy seems to be a good option for treating and preventing a coccygeal pressure ulcer for individuals with a SCI.

A limitation of this retrospective follow-up single center cohort study is the lack of a control group. In addition, geriatric individuals with multiple accompanying co-morbidities are included within the study population, increasing the risk of relapse.

Due to the high mortality rate of these individuals within 5 years after coccygectomy (19\%), less data could be collected after 5 years. The strengths of this study include the high number of individuals included and the long observation period. In addition, all surgeries were performed by a single surgeon, increasing comparability. The follow-up treatment of all individuals also proceeded according to a standardized procedure and was the same for all individuals. To improve on this data, further studies in specialized centers for the evaluation of the success of therapy are needed.

\section{Conclusion}

The coccygectomy in addition to flap-based plastic surgery procedures in a category 3 and 4 of a coccygeal pressure ulcer is an adequate method for treatment and prevention of recurrence in individuals with SCI or other neurological disease with similar clinical symptoms.

However, the best method to prevent a pressure ulcer in general is to avoid it in first line! However, this needs structured information about prevention of pressure ulcers for individuals with an SCI from beginning on and especially during the rehabilitation. Self-check of the skin in the pelvic region, and regular load reliefs or weight shifts, are just a few important key points. Also, regular checkup for outpatients can ensure that an early identification and correct treatment in a rehabilitation unit can prevent a surgical intervention. An upright sitting position in the wheelchair, monitoring of seat cushions, and avoiding sitting in a beach-chair position are all practices that are of the utmost importance for the prevention of a coccygeal pressure ulcer.

\section{Data availability}

Original data are stored at the REHAB Basel, Centre for Neurorehabilitation and Paraplegiology.

Acknowledgements We would like to thank RR and the REHAB Basel for the opportunity to perform the study, all the contributors and GNJ for all of his support.

\section{Compliance with ethical standards}

Conflict of interest The authors declare that they have no conflict of interest.

Publisher's note Springer Nature remains neutral with regard to jurisdictional claims in published maps and institutional affiliations. 


\section{References}

1. Lüscher NJ, Zäch GA, Mäder M, Urwyler A. Dekubitusinzidenz bei frischer Querschnittlähmung. Rehabilitation beginnt am Unfallort. Berlin: Springer; 1992.

2. DeJong G, Tian W, Hsieh CH, Junn Ch, Karam Ch, Ballard P, et al. Rehospitalization in the first year of traumatic spinal cord injury after discharge from medical rehabilitation. Arch Phys Med Rehabil. 2013;94:87-97.

3. Tadiparthi S, Hartley A, Alzweri L, Mecci M, Siddiqui H. Improving outcomes following reconstruction of pressure sores in spinal injury patients: a multidisciplinary approach. J Plast Reconstr Aesthet Surg. 2016;69:994-1002.

4. White BA, Dea N, Street JT, L Cheng Ch, Rivers C, Attabib N, et al. The economic burden of urinary tract infection and pressure ulceration in acute traumatic spinal cord injury admissions: evidence for comparative economics and decision analytics from a matched case-control study. J Neurotrauma. 2017;34:2892-2900.

5. Kruger EA, Pires M, Ngann Y, Sterling M, Rubayi S. Comprehensive management of pressure ulcers in spinal cord injury: current concepts and future trends. J Spinal Cord Med. 2013;36:572-85.

6. Liakoni E. Prädilektionsstellen für decubitus bei querschnittlähmung und ihre abhänigkeit vom neurologischen lähmungsmuster. Dissertation. Schweiz: Basel; 2010.

7. Committee Membership, Burns S, Biering-Sørensen F, Donovan W, Graves D, Jha A, Johansen M, et al. International standards for neurological classification of spinal cord injury, revised 2011. Top Spinal Cord Inj Rehabil. 2020;18: 85-99. https://doi.org/10.1310/ sci1801-85.

8. De Roche R. Störfall decubitus: handbuch zur gesundsheitsökonomischen bedeutung, prävention, konservativen und chirurgischen therapie. Schweiz: Basel; REHAB Basel; 2012. ISBN 978-3-033-03440-2.

9. Grassi R, Lombardi G, Reginelli A, Capasso F, Romano F, Floriani I, et al. Coccygeal movement: assessment with dynamic MRI. Eur J Radiol. 2007;61:473-9.

10. Fu J, Jones M, Jan YK. Development of intelligent model for personalized guidance on wheelchair tilt and recline usage for people with spinal cord injury: methodology and preliminary report. J Rehabil Res Dev. 2014;51:775-88.

11. Duncan KD. Preventing pressure ulcers: the goal is zero. Jt Comm J Qual Patient Saf. 2007;33:605-10.

12. Provodin O, Lüscher NJ. Dekubitalulcera über dem Os coccygis, pathophysiologie und chirurgische behandlung. Dissertation Kantonspital Basel. 1993. Provodin O, Schweiz: Basel.

13. Doursounian L, Maigne JY, Cherrier B, Pacanowski J. Prevention of post-coccygectomy infection in a series of 136 coccygectomies. Int Orthop. 2011;35:877-81.

14. Benditz A, König MA. Therapieresistente Kokzygodynie sollte nicht länger als mythos angesehen werden. Der Orthopäde. 2019;48:92-5.

15. Nathan ST, Fisher BE, Robert CS. Coccydynia: a review of pathoanatomy, aetiology, treatment and outcome. J Bone Jt Surg Br Vol. 2010;92:1622-7.

16. Antoniadis A, Ulrich NHB, Senyurt H. Coccygectomy as a surgical option in the treatment of chronic traumatic coccygodynia: a single-center experience and literature review. Asian Spine J. 2014;8:705.

17. Kerr EE, Benson D, Schrot RJ. Coccygectomy for chronic refractory coccygodynia: clinical case series and literature review. J Neurosurg: Spine. 2011;14:654-63.

18. Grgić V. Coccygodynia: etiology, pathogenesis, clinical characteristics, diagnosis and therapy. Lijec Vjesn. 2012;134:49-55.

19. Grosso NP. Total coccygectomy for the relief of coccygodynia: a retrospective review. J Spinal Disord. 1995;8:328-30.

20. Maigne JY, Lagauche D, Doursounian L. Instability of the coccyx in coccydynia. J Bone Jt Surg Br Vol. 2000;82:1038-41.

21. Lüscher NJ, Krupp S, Kuhn W, Zäch GA. The sensory tensor fasciae latae flap: a 9-year follow-up. Ann Plast Surg. 1991;26:306-10.

22. Wanner MB, de Roche R, Lüscher NJ. Chirurgische therapie des decubitus. Swiss Med. 1995;17:73-9.

23. Langauer S. Dekubitus beim geriatrischen patienten: stellenwert, indikationen und resultate der chirurgisch-neurologischen lähmungsmuster. Dissertation. 1994. Schweiz: Universität Basel; 1994.

24. Lüscher NJ. Dekubitalulzera der Beckenregion: diagnostik und chirurgische therapie. Aktuelle Probl Chir Orthop. 1989;33. 